\title{
Proinflammatory cytokines differentially influence adult hippocampal cell proliferation depending upon the route and chronicity of administration
}

\author{
Julie Anne Seguin \\ Jordan Brennan \\ Emily Mangano \\ Shawn Hayley
}

Institute of Neuroscience, Carleton University, Ottawa, Ontario, Canada

Correspondence: Shawn Hayley I 25 Colonel By Drive, Ottawa, Ontario, Canada KIS 5B6

$\mathrm{Tel}+\mathrm{I} 6135202600$ Ext 6314

$\mathrm{Fax}+\mathrm{I} 6135204052$

Email shayley@ccs.carleton.ca

\begin{abstract}
Disturbances of hippocampal plasticity, including impaired dendritic branching and reductions of neurogenesis, are provoked by stressful insults and may occur in depression. Although corticoids likely contribute to stressor-induced reductions of neurogenesis, other signaling messengers, including pro-inflammatory cytokines might also be involved. Accordingly, the present investigation assessed whether three proinflammatory cytokines, namely interleukin-1 $\beta$ (IL-1 $\beta$ ), IL-6, and tumor necrosis factor- $\alpha$ (TNF- $\alpha$ ) (associated with depression) influenced cellular proliferation within the hippocampus. In this regard, systemic administration of TNF- $\alpha$ reduced 5-bromo-2-deoxyuridine (BrdU) labeling within the hippocampus, whereas IL-1 $\beta$ and IL-6 had no such effect. However, repeated but not a single intra-hippocampal infusion of IL-6 and IL-1 $\beta$ actually increased cellular proliferation and IL-6 infusion also enhanced microglial staining within the hippocampus. Yet, no changes in doublecortin expression were apparent, suggesting that the cytokine did not influence the birth of cells destined to become neurons. Essentially, the route of administration and chronicity of cytokine administration had a marked influence upon the nature of hippocampal alterations provoked, suggesting that cytokines may differentially regulate hippocampal plasticity in neuropsychiatric conditions.
\end{abstract}

Keywords: cytokine, depression, neuroplasticity, hippocampus, stressor

\section{Introduction}

Recent efforts assessing the mechanisms of depression have focused on the possibility that immune factors, particularly cytokines, may contribute to this disorder. Besides being responsible for communication between immune cells, these messengers also act as immunotransmitters, alerting the central nervous system of immunological insults. ${ }^{1-4}$ In this regard, the proinflammatory cytokines, interleukin-1 $\beta$ (IL-1 $\beta$ ) and tumor necrosis factor- $\alpha$ (TNF- $\alpha$ ), and IL-6 to a lesser extent, stimulated hypothalamic-pituitary-adrenal (HPA) activity and augmented turnover of serotonin (5-HT) and norepineprine (NE) in several stressor-sensitive hypothalamic and extrahypothalamic brain regions..$^{5-7}$ Moreover, an IL-1 receptor antagonist attenuated the neurochemical effects of a restraint stressor, ${ }^{8}$ suggesting that the stressor was acting through cytokine dependent mechanisms. Beyond these effects, IL-1 $\beta$ and TNF- $\alpha$ induced behavioural alterations (eg, anhedonia) that were reminiscent of the effects of stressors as well as those that are characteristic of depressive disorder, ${ }^{2,9-11}$ For instance, social exploration and responding for a palatable solution were significantly decreased in rodents that received peripheral or central IL-1 $\beta$ administration. ${ }^{12-14}$

In addition to their neurochemical effects, cytokines, like stressors, may also influence processes important for neuroplasticity, and hence may influence cognitive and affective functioning. In fact, IL-1 $\beta$ and TNF- $\alpha$ were reported to alter hippocampal long-term potentiation and together with IL-6, also influenced dendritic branching 
of hippocampal neurons. ${ }^{15}$ Similarly, depressed individuals were reported to display elevated levels of these cytokines along with cognitive deficits, which in some cases may be coupled with reductions of the growth factor, brain-derived neurotrophic factor (BDNF), as well as decreased hippocampal volume. ${ }^{16-20}$

Impairment in the generation of new hippocampal cells has been implicated in the altered hippocampal plasticity observed in depressed subjects and animals exposed to various stressors. ${ }^{21-24}$ The impact of stressful challenges upon neurogenesis or gliogenesis appears to vary with the type of stressor and chronicity of exposure. Indeed, chronic but not acute restraint reduced the proliferation of newly born dentate gyrus cells, ${ }^{25}$ however, an acute psychosocial stressor did reduce the survival of recently generated hippocampal cells. ${ }^{26}$ Importantly, disturbances of neurogenesis may have functional implications for depression, as indicated by the finding that the behavioral effects of the antidepressants, fluoxetine and imiprimine, were ameliorated by selective impairment of hippocampal neurogenesis. ${ }^{27}$

Although it is clear that glucocorticoid elevations associated with depression and stressor exposure can impair the birth of hippocampal cells, ${ }^{20,28,29}$ pro-inflammatory cytokines may also contribute in this regard. Indeed, using a dosing schedule similar to that used clinically to treat cancer and viral infection and which also elicits symptoms of depression, administration of interferon- $\alpha$ (IFN- $\alpha$ ) reduced hippocampal neurogenesis in rodents, and this effect was dependent upon endogenous IL- $1 \beta$ levels. ${ }^{30}$ In agreement with this finding, the reduction of hippocampal neurogenesis normally associated with chronic stressor exposure was prevented in IL-1 type 1 receptor knockout mice. ${ }^{31}$ Likewise, alterations of hippocampal neurogenesis were reported in adult transgenic mice over-expressing IL- $6,{ }^{31}$ and administration of the bacterial endotoxin lipopolysaccharide (LPS) suppressed neurogenesis while concomitantly elevating IL-1 $\beta$ and TNF- $\alpha .{ }^{32}$ The effects of LPS on neurogenesis were reversed by the tetracycline derivative, minocycline, or the nonsteroidal anti-inflammatory compound, indomethacin, both of which are potent inhibitors of inflammatory cytokines and microglia. ${ }^{32,33}$

Cytokines may influence neuroplasticity through actions upon peripheral sites, such as vagal afferents, receptors at the blood brain barrier or immune cells, or by directly acting within the central nervous system (CNS). ${ }^{34-36}$ Hence, the current study assessed the impact of systemic and intra-hippocampal infusion of the cytokines, IL-1 $\beta$, IL-6 and TNF- $\alpha$ upon the generation of new dentate gyrus cells. Since the chronicity of stressor exposure greatly influences the nature of neuroplastic effects imparted by such challenges, ${ }^{25}$ it was also of interest to evaluate whether the effects the cytokines were augmented or diminished with repeated infusion. Presently, we report that systemic TNF- $\alpha$ dramatically reduced the generation of new dentate gyrus cells that incorporated the cellular proliferation marker, 5-bromo-2-deoxyuridine (BrdU), whereas infusion of the cytokine directly into the hippocampus had no such effect. In contrast, repeated but not a single intra-hippocampal infusion of IL-6 induced a profound elevation of hippocampal cells expressing BrdU, together with enhanced microglial reactivity. Thus, a single TNF- $\alpha$ exposure may negatively influence the production of hippocampal cells by affecting peripheral processes, whereas repeatedly elevated IL-6 may act within the hippocampus to promote cellular proliferation. However, since no changes in immunoreactivity for the immature neuronal marker, doublecortin, were evident following the treatments, it appears that the cytokines did not influence the rate of neurogenesis. These data suggest that cytokine alterations provoked by stressful or inflammatory challenges may influence hippocampal plasticity and that such effects may vary according to the specific cytokine(s) involved, as well as the physiological region and chronicity of exposure.

\section{Methods}

\section{Animals}

Each of the two experiments involved naïve, male CD-1 mice obtained from Charles River Canada (Laprairie, Quebec) at 8-10 weeks of age. Mice were permitted 2-3 weeks to acclimatize to the laboratory before the start of each experiment. Mice were housed in groups of four in standard $(27 \times 21 \times 14 \mathrm{~cm})$ polypropylene cages and transferred to individual housing 1 week before the initiation of a study. Animals were kept on a 12-h light-dark cycle (lights on: 0800-2000 hours) in a temperature-controlled vivarium $\left(22-25^{\circ} \mathrm{C}\right)$, and were provided free access to mouse chow and water. All experimental procedures complied with the current guidelines stipulated by the Canadian Council on Animal Care and were approved by the Carleton University Animal Care Committee.

\section{Surgery and cytokine treatments} Experiment I

It was of interest to determine the effect of systemic proinflammatory cytokine administration upon hippocampal 
neuroplasticity. To this end, animals ( $n=8 /$ group) received intraperitoneal (i.p.) administration of either IL-1 $\beta(0.1 \mu \mathrm{g})$, IL-6 $(1.0 \mu \mathrm{g})$, TNF- $\alpha(1.0 \mu \mathrm{g})$ or vehicle. In order to later examine and quantify any changes in cellular proliferation, animals were injected with 5-bromo-2-deoxyuridine (BrdU), an exogenous cell proliferation agent widely used to label mitotically active cells. Specifically, mice received one i.p. injection of BrdU (200 mg/kg; Sigma-Aldrich, Toronto, $\mathrm{ON}$ ) dissolved in $0.9 \%$ saline immediately prior to the cytokine or vehicle injection.

\section{Experiment 2}

This experiment assessed whether cytokines influence cellular proliferation by directly acting within the hippocampus, as opposed to activating peripheral immune substrates, which in turn, influence CNS processes. Thus, animals were stereotaxically implanted with a 33 gauge guide cannula just above the hippocampus (placement coordinates: $\mathrm{A} / \mathrm{P}=-1.82 \mathrm{~mm}, \mathrm{D} / \mathrm{V}=-1.25 \mathrm{~mm}, \mathrm{M} / \mathrm{L}= \pm 1.00 \mathrm{~mm}$ ). Following a four day recovery period, mice $(n=6$ /group) were subjected to either a single infusion or five days of once-daily infusions of saline, IL- $1 \beta(0.01 \mu \mathrm{g})$, IL-6 $(0.05 \mu \mathrm{g})$ or TNF- $\alpha(0.05 \mu \mathrm{g})$. Cytokines were dissolved in vehicle and in all cases $2 \mu \mathrm{L}$ of solution was slowly infused over a period of five minutes using a Pico Plus syringe pump (Harvard Apparatus Inc., Holliston, MA). In order to label dividing cells, animals received one i.p. injection of BrdU $(200 \mathrm{mg} / \mathrm{kg})$ immediately prior to the final cytokine or saline injection.

\section{Tissue preparation}

At 24 hours after all cytokine or vehicle injections, animals were overdosed with pentobarbital and transcardially perfused with $0.9 \%$ saline, followed by $4 \%$ paraformaldehyde (PFA). Brains were collected, postfixed for 24 hours in PFA and then cryoprotected, for at least 3 days, in a $20 \%$ sucrose solution with $0.02 \%$ azide. Using a cryostat, $20-\mu \mathrm{m}$ thick coronal hippocampal sections were collected and mounted on gelatine-coated slides. Ten slides were collected (6 sections/slide) from each brain and stored at $-80{ }^{\circ} \mathrm{C}$ pending analysis. Hippocampal levels collected were operationally defined as early/rostral (bregma -1.22 to -1.82 ), middle (bregma -1.82 to -2.46 ) and late/caudal (bregma -2.46 to -2.92 ). Thus, data analyses assessed whether cytokine treatments preferentially affected proliferation at specific anatomical levels of the hippocampal dentate gyrus. In all cases, cannula placement was verified using cresyl violet staining.

\section{Immunohistochemistry and quantification procedures}

Brain sections were processed for BrdU labeling by incubation in $1 \mathrm{~N} \mathrm{HCl}\left(2\right.$ hours at $\left.50^{\circ} \mathrm{C}\right)$ to denature DNA, followed by neutralization with $0.1 \mathrm{M}$ borate buffer $(\mathrm{pH} 8.5)$ for ten minutes. After rinsing with PBS $(0.01 \mathrm{M})$, the sections were incubated with mouse monoclonal anti-BrdU (1:200 Sigma-Aldrich, Toronto, ON) for 48 hours at $4{ }^{\circ} \mathrm{C}$. Thereafter, CY3 conjugated donkey anti-mouse (1:200; Jackson ImmunoResearch Laboratories Inc., PA) antiserum was used as the secondary antibody. Separate section were also processed in order to quantify whether changes in BrdU labeling reflect variations of neurogenesis, as indicated by a transiently expressed marker of immature neurons, doublecortin (DCX). Thus, sections were incubated with anti-DCX (1:600; guinea pig polyclonal, Abcam, FL) for 24 hours at $4{ }^{\circ} \mathrm{C}$ followed by anti-rabbit CY3 (1:200; Goat polyclonal to Guinea Pig, Abcam, FL) for one hour at room temperature.

Markers for astrocytes and microglia were also used in order to determine whether the cytokines influenced glial activity, such that sections were first incubated with antiGFAP (1:500, mouse polyclonal, Chemicon International) or anti-CD11b (1:1000; rat monoclonal, Raleigh, NC) for 24 hours at $4{ }^{\circ} \mathrm{C}$. Subsequently, the sections were incubated with CY3 conjugated donkey anti-mouse or ant-rat (1:200, Jackson Immunoresearch) antibodies for 2 hours at room temperature.

All quantitative analyses were performed under conditions in which the rater was blind to the treatments received. For Experiment 1, the total number of bilaterally labeled BrdU-positive cells was counted within the rostral, middle and caudal portions of the dentate gyrus. Using 40X magnification, the $20 \mu \mathrm{m}$ tissue sections were counted and the average number of immuno-positive cells per section calculated for each hippocampal level. For Experiment 2, the number of BrdU-positive cells of the ipsilaterally infused hemisphere was assessed.

\section{Statistics}

Data from Experiment 1 were analyzed at each hippocampal level using one-way ANOVAs, with IL-1 $\beta$, IL-6, TNF- $\alpha$, and control as factor levels for the treatment condition. Data for Experiment 2 were analyzed at each hippocampal level using two-way ANOVAs, with chronicity (acute and sub-chronic) and treatment (IL-1 $\beta$, IL-6, TNF- $\alpha$, and control) as factors. Post hoc Tukey tests were performed to determine specific between-group differences $(\alpha=0.05)$. 


\section{Results}

Experiment I: Acute systemic cytokine exposure alters hippocampal cell proliferation (BrdU) and neurogenesis (DCX)

Although no significant effects were observed at the middle and caudal hippocampal levels, an ANOVA did reveal that the cytokine treatments provoked significant differences in $\mathrm{BrdU}$ counts at the rostral level of the hippocampus $F(3,23)=3.02$, $p<0.05$. Post hoc Tukey comparisons indicated that mice of the TNF- $\alpha$ group differed significantly from saline-injected animals $(p<.05)$. Indeed, as shown in Figure 1, mice that received i.p. administration of TNF- $\alpha$ had an approximately sevenfold reduction of BrdU labelling in the rostral portion of the dentate gyrus relative to those that received saline (Figure 1). Although IL-6 treatment seemed to reduce the number of proliferating cells, the observed results were relatively variable, precluding the detection of statistically significant differences. With respect to the morphological
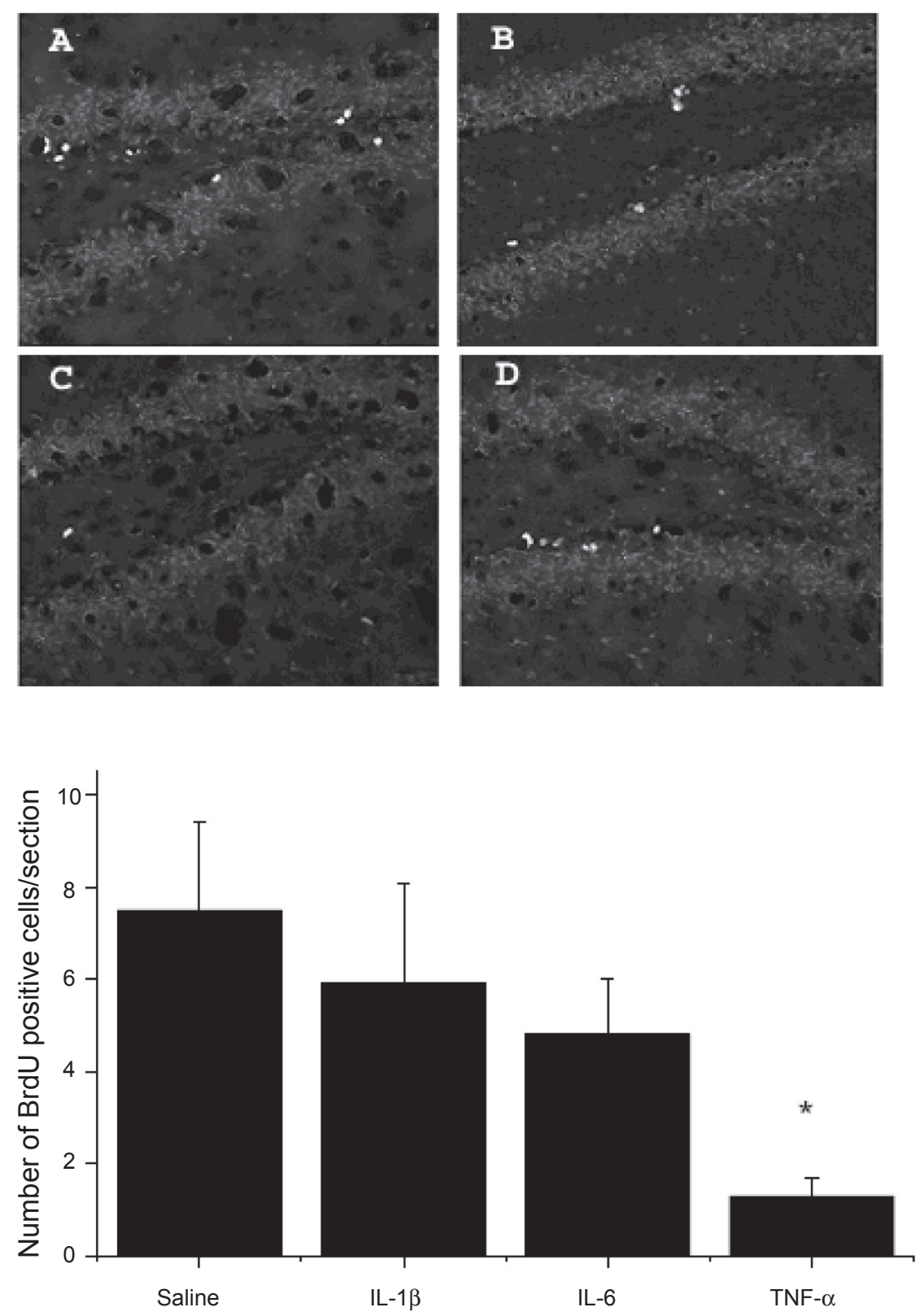

Figure I BrdU immunopositive cells are depicted in the photomicrographs of the rostral portion of hippocampal dentate gyrus of animals subjected to i.p. injection of A) saline, B) IL-I $\beta(0.1 \mu \mathrm{g})$, C) TNF- $\alpha(1.0 \mu \mathrm{g})$ or (d) IL-6 (I.0 $\mu \mathrm{g})$. There was a clear reduction of BrdU-labelled cells in mice that received TNF- $\alpha$ (panel C), relative to saline injected controls (panel A). The bottom bar graph displays the mean ( \pm SEM) number of BrdU-positive cells per section and confirms the suppression of BrdU labeling following systemic administration of TNF- $\alpha$.

Notes: ${ }^{p} p<0.05$ vs. saline-treated animals, $10 \times$ magnification. 
aspects of the proliferating cells, BrdU-positive cells were generally small, oval-shaped and had a stippled pattern. They were mainly in the subgranular zone (SGZ) of the dentate gyrus and the hilus, often in clusters of two or three. These morphological characteristics and pattern of distribution closely resemble that ordinarily reported. ${ }^{37}$ Although no significant treatment effects were observed in the middle and caudal levels of the hippocampus, there was a trend towards a cytokine-induced reduction of BrdU labelling, such that in the middle hippocampal region, IL- $1 \beta$ and TNF- $\alpha$ provoked a modest decrease in BrdU staining (35\%-40\% decrease) relative to saline treated mice (data not shown).

In contrast to the effects of TNF- $\alpha$ upon hippocampal BrdU incorporation, the cytokine treatments did not significantly influence expression of the immature neuronal marker, DCX, within the rostral, middle or caudal regions of the dentate gyrus $F_{S}(3,23)<1$. For example, as shown in Figure 2, DCX immunoreactivity within the rostral dentate gyrus did not differ between mice that received either systemic TNF- $\alpha$ or vehicle.

\section{Experiment 2: A single and repeated central cytokine infusion differentially influence hippocampal cell proliferation (BrdU, CDIIb)}

Significant variations of BrdU labelling were evident at each of the three hippocampal levels assessed. Indeed, at the most rostral level of the dentate gyrus BrdU labelling varied as a function of the interaction between intra-hippocampal cytokine infusion and chronicity of administration, $F(3,38)=3.49, p<0.05$. The follow up Tukey tests revealed that the repeated, but not single, intra-hippocampal IL-6 infusion significantly increased the hippocampal BrdU labelling, relative to saline infused mice ( $\mathrm{p}<0.05$; Figure 3$)$. Although the interaction failed to reach significance at the
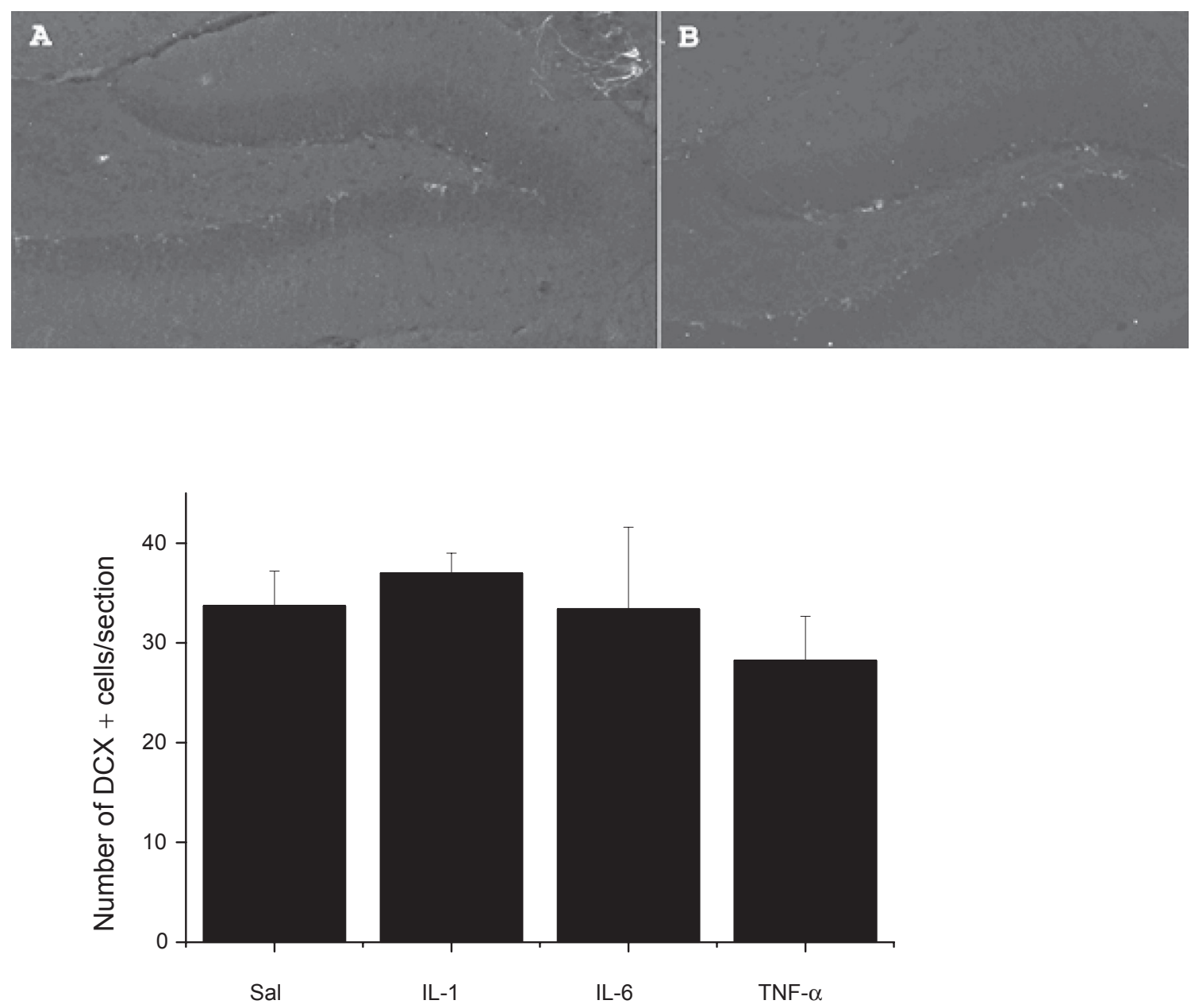

Figure 2 The photomicrographs ( $4 \times$ magnification) show doublecortin (DCX) positive immature neurons within the dentate gyrus of the rostral portion of dorsal hippocampus. There was no significant difference in DCX expression between mice that received i.p. saline $\mathbf{A}$ ) or those treated with i.p. TNF- $\alpha$ B) The inset (upper right corner of panel A) reveals a higher magnification $(20 \times)$ image of the DCX positive soma and projections from a saline-treated animal. 

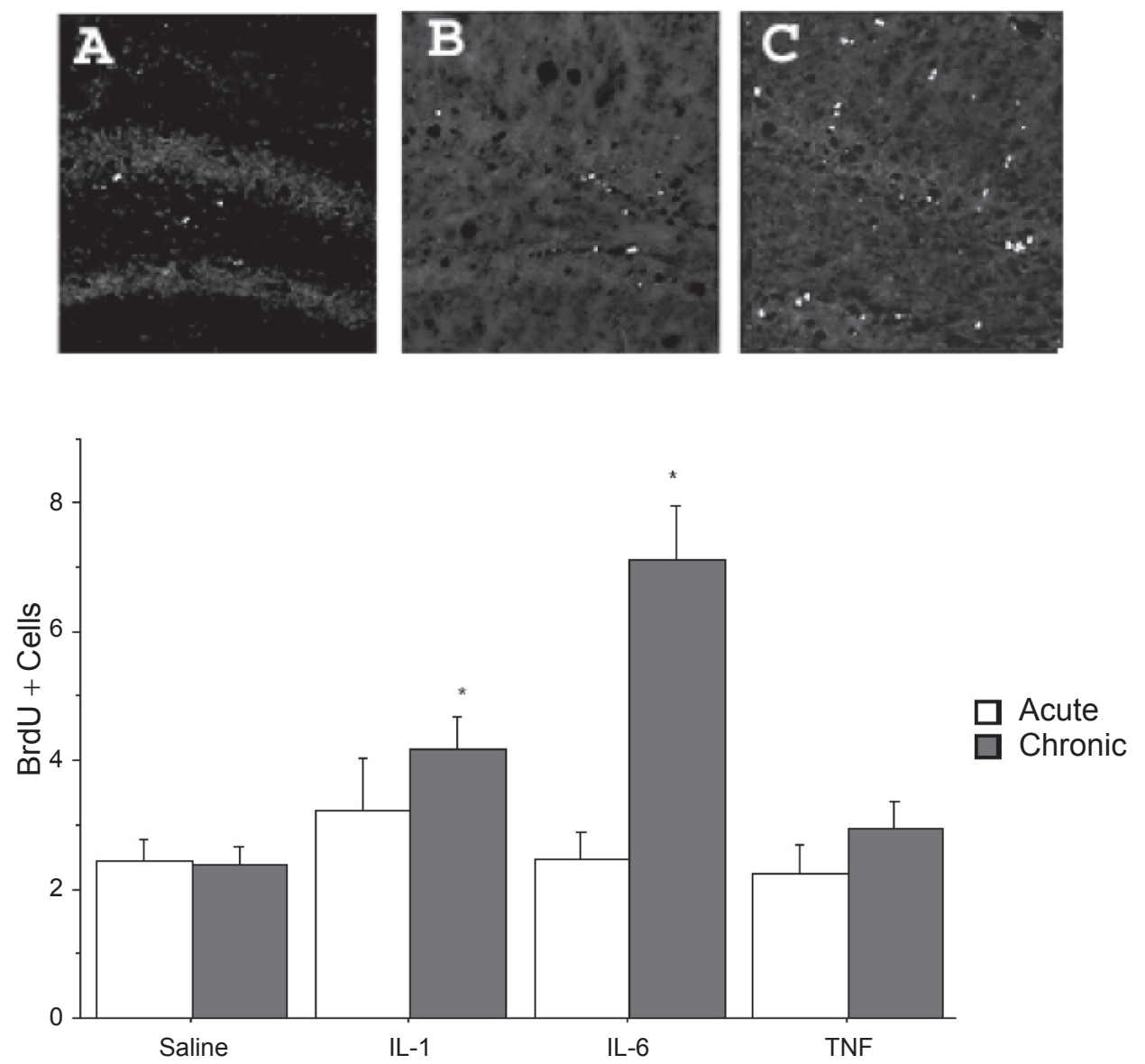

Figure 3 The photomicrographs depict BrdU-positive cells within the dentate gyrus following a single or repeated intra-hippocampal cytokine or saline infusion. Elevated BrdU staining within the dentate gyrus was observed in animals subjected to repeated intra-hippocampal infusion of either IL-I $\beta$ (panel B) or IL-6 (panel C), relative to animals that received vehicle (panel A). The BrdU increase was particularly robust for the repeated IL-6 treatment. Quantification of mean ( \pm SEM ) number of BrdU-positive cells per section confirmed that repeated (grey bars) but not a single (white bars) intra-hippocampal infusion of IL-I $\beta$ and IL-6 significantly increased BrdU staining, relative to animals that received infusion of saline.

Notes: ${ }^{*} p<0.05$ vs saline-treated animals, 10x magnification.

middle hippocampal level, the ANOVAs for the individual cytokine infusion and chronicity main effects were significant, Fs $(3,38)=4.31,(1,38)=3.77$, respectively, $\mathrm{p}<0.05$. The post hoc analyses revealed that IL-1 $\beta$ and IL-6 infusion increased the number of BrdU positive cells relative to the respective saline treated animals and that repeated cytokine administration enhanced levels of the proliferation marker above that observed following a single acute treatment $(\mathrm{p}<0.05)$. Finally, ANOVA of the caudal portion of the dentate gyrus revealed that BrdU immunoreactivity varied as a function of the cytokine infusion and chronicity of administration $F(3,38)=3.23, p<0.05$. As shown in Figure 3, follow up analyses of the significant interaction indicated that repeated infusion of IL- 6 increased the number of BrdU positive cells at this level of the hippocampus, relative to the remaining groups $(\mathrm{p}<0.05)$. Of particular interest was the fact that repeated IL-6 infusion had the greatest impact on cell proliferation and this was evident at all three levels of the dentate gyrus. Importantly, BrdU levels were identical in animals that received either the single or repeated intrahippocampal saline infusion, suggesting that repeated vehicle infusion alone did not affect cellular proliferation. Surprisingly, intra-hippocampal infusion of TNF- $\alpha$ failed to influence BrdU labelling, suggesting that the BrdU reduction observed in mice that received i.p. TNF- $\alpha$ in Experiment 1 may stem from actions of the cytokine at peripheral sites. The lack of an effect of TNF- $\alpha$ on BrdU incorporation may be related to the timing of sacrifice or dose of the cytokine administered. Indeed, one report did indicate that central TNF- $\alpha$ infusion increased BrdU labelling; however, this study used a substantially higher dose of the cytokine ( $355 \mathrm{ng}$ vs $50 \mathrm{ng}$ used in the present study) and involved an intra-ventricular rather than intra-hippocampal route of delivery. ${ }^{59}$

As in the previous experiment, the BrdU-positive cells were located mainly in small clusters in the subgranular and the hilus regions of the dentate gyrus (Figure 3). 
However, some BrdU labelling was also observed just above the dentate gyrus and around the CA1 region of the hippocampus, possibly reflecting migration of newly born neurons or alternatively, these cells may be of glial origin. Indeed, paralleling the BrdU variations, repeated but not a single IL-6 infusion provoked intense immunoreactivity for the microglial marker, CD11b, within the CA1 hippocampal region, directly above the granule cell layer of the dentate gyrus (Figure 4). In fact, a wave of CD11b labelling was apparent from the cannula tract into the corpus callosum and the hippocampus, suggesting that repeated IL-6 exposure activated microglial proliferation. In contrast, no significant variations of staining the astrocyte marker, GFAP, or the immature neuronal marker, DCX, were evident among the treatment groups (data not shown). Thus, IL-6 appeared to be selectively affected microglia and not immature neurons or astrocytes.

\section{Discussion}

The present findings are consistent with the proposition that variations of cytokines may impair hippocampal plasticity. In fact, we and others have previously posited that alterations of hippocampal volume or neuroplasticity often associated with depression may stem from stressor or cytokine induced disturbances of cellular proliferation, as well as other morphological abnormalities, such as reduced dendritic branching. ${ }^{2,21,23,24}$ Moreover, impairment of hippocampal cellular proliferation may have functional implications for
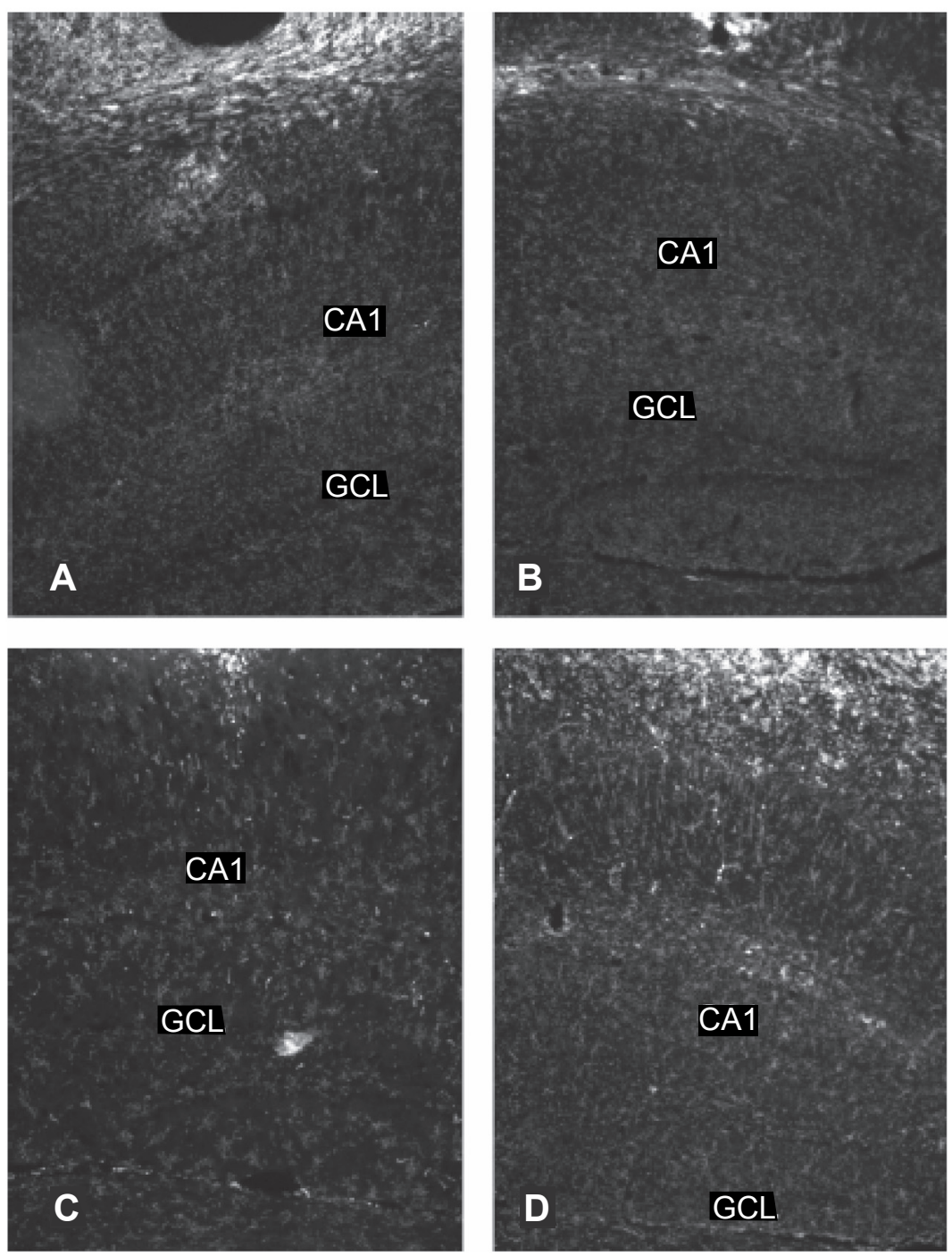

Figure 4 Photomicrographs of labeling for the microglial marker, CDI I b, in animals subjected to a single or repeated intra-hippocampal infusions of saline or IL-6. The CDI I b immunoreactivity was evident in the granule cell layer $(\mathrm{GCL})$ of the dentate gyrus, as well as within the CAI hippocampal region. Single $(A)$ and repeated $(B)$ infusion of saline provoked minimal CDI Ib staining in the GCL and CAI.Although the single IL-6 infusion (C) induced modest CDI Ib immunoreactivity, repeated infusion of the cytokine (D) produced the most marked increase of expression of the microglial marker within the GCL and CAI areas of the hippocampus. $4 \times$ magnification. 
depression, especially as the efficacy of antidepressants was reduced in the face of impaired hippocampal neurogenesis. ${ }^{27}$ Yet, surprisingly little is known of the potential mechanisms through which stressors or other factors aligned with depression may impact upon hippocampal cellular proliferation.

In addition to their role in depressive-like pathology, 2,9,11 pro-inflammatory cytokines might influence neurogenesis. Indeed, when administered chronically (in a schedule mimicking an immunotherapeutic protocol), IFN- $\alpha$ elicited a marked reduction of hippocampal neurogenesis. ${ }^{30}$ Interestingly, the impact of IFN- $\alpha$ upon neurogenesis was ameliorated by central treatment with the cytokine antagonist, IL-1Ra, suggesting that endogenous IL- $1 \beta$ was an important factor inhibiting neurogenesis..$^{30}$ Furthermore, IL-1 receptor knockout prevented the reduction of neurogenesis associated with a chronic stress regimen ${ }^{51}$ and attenuation of the inflammatory response using indomethacin likewise normalized the reduction of neurogenesis associated with endotoxin treatment or irradiation. ${ }^{32}$ Thus, there is reason to believe that inflammatory cytokines may be involved in regulating neuroplastic mechanisms aligned with neurogenesis.

\section{Systemic cytokine administration and hippocampal cellular proliferation}

Presently, we report a marked reduction of cell proliferation, as indicated by BrdU incorporation, within the rostral portion of the hippocampal dentate gyrus (DG) following systemic administration of TNF- $\alpha$. In contrast, the cytokine did not affect hippocampal DCX expression, suggesting that the variations of BrdU labelling reflecting changes in the proliferation of non-neuronal cells, these could likely be glia given their marked sensitivity to cytokine signalling. Interestingly, intra-hippocampal TNF- $\alpha$ infusion did not significantly affect hippocampal cellular proliferation. Thus, the peripheral rather than central actions of TNF- $\alpha$ may be most pertinent in affecting the birth of new DG cells. This is consistent with our previous findings that systemic TNF- $\alpha$ elicited a marked elevation of corticosterone and sensitized mice to the impact of subsequent exposure to the cytokine, whereas central infusion of the cytokine had only modest actions upon hormone levels and did not provoke such a sensitization. $^{5}$

We have previously found that IL-1 $\beta$ (even at 4 fold lower doses) generally produces more profound behavioral, neuroendocrine and neurotransmitter effects than does TNF- $\alpha .^{38,39}$ However, since the systemic doses of TNF- $\alpha$ that were used in the present study were 10-fold higher than those of IL-1 $\beta$, it is not entirely surprising that the former cytokine had more potent actions on cell proliferation. Moreover, TNF- $\alpha$ has a more prominent role than IL- $1 \beta$ in a number of inflammatory immune processes, including acute phase reactions and T and NK cell cytotoxicity. ${ }^{40}$ As well, TNF- $\alpha$ may have influenced hippocampal cellular survival by activating apoptotic cascades. ${ }^{41}$ Interestingly, in fact, the two TNF-a receptors, TNFR-1 (which possess a caspaselinked intra-cellular death domain) and TNFR-2, appear to differentially influence neurogenesis, such that inhibition of the former enhanced hippocampal neurogenesis, whereas ablation of TNFR-2 reduced neurogenesis. ${ }^{42}$ Thus, the more potent effects of TNF- $\alpha$ on cell proliferation may stem from multiple immunological, hormonal or apoptotic processes associated with the cytokine. However, since only a single dose of each cytokine was used, taken together with the possibility that differing dose-response relationships might exist between different cytokines, ${ }^{43}$ it may be that other doses of IL-1 $\beta$ or IL- 6 would exert more marked effects on cellular proliferation.

There are several potential routes through which the systemically injected cytokines might have influenced hippocampal cellular proliferation. Although cytokines do not readily penetrate the blood-brain barrier $(\mathrm{BBB})$ (owing to their size and charge), they can cross at "leaky" BB regions, including the median eminence region of the hypothalamus, as well as other circumventricular sites, including the subfornical region. ${ }^{54,55}$ Specific carrier mediate transport mechanisms have also been reported to transport certain cytokines across the $\mathrm{BBB}^{56}$ and cytokine administration itself (eg, TNF- $\alpha$ ) may disrupt BBB integrity. ${ }^{57}$ As well, several cytokines were reported to trigger the transcription of genes at cells of the BBB including NF- $\kappa \mathrm{B}$ and COX-2, and these factors then promoted signaling within the CNS. ${ }^{58}$ Hence, any of these potential routes might explain how the peripheral cytokine treatments altered hippocampal BrdU expression.

\section{Central cytokine infusion and hippocampal cellular proliferation}

In contrast to the effects of systemic TNF- $\alpha$, repeated intrahippocampal infusion of IL- 6 and to a lesser degree IL-1 $\beta$, increased the number of BrdU-positive cells in the dentate gyrus, suggesting possible pro-mitotic effects of these cytokines. It is unclear why intra-hippocampal cytokine infusion had consequences so remarkably different from that observed following systemic administration. It may be that sufficiently high concentrations of the cytokines did not reach the hippocampus following their systemic administration. The fact that repeated but not a single intra-hippocampal IL-6 
infusion markedly elevated BrdU staining suggests that the passage of time following introduction of the cytokine may be fundamental to the cytokine's actions or alternatively, that a single infusion alone was not a sufficiently potent challenge to influence hippocampal cellular proliferation. It is possible that IL-6 may have promoted cellular proliferation by enhancing the production of growth factors from microglia or astrocytes, ${ }^{44}$ particularly given that IL-6 elevated expression of the microglial marker, CD11b, within the hippocampus.

Consistent with the IL-6-induced changes in cell proliferation, several studies indicated that this cytokine influenced the survival, proliferation and differentiation of a variety of CNS cells. ${ }^{45-48}$ These diverse actions appeared to be cellspecific and dependent upon the particular experimental preparation. In fact, while Vallières and colleagues reported a suppression of hippocampal neurogenesis in adult transgenic mice over-expressing IL-6, the cytokine actually enhanced astrogliogenesis, microgliosis and oligodendrogenesis. ${ }^{31}$ These reports, together with the enhanced CD11b staining we observed in the DG, suggest that the elevated BrdU staining observed following repeated IL-6 infusion may reflect augmented gliogenesis rather than neurogenesis. However, given that IL-6 receptors are found on DG endothelial cells, ${ }^{52}$ coupled with findings showing that a large proportion of diving DG cells express endothelial markers and that IL-6 enhanced the in vitro proliferation of endothelial cells, ${ }^{53}$ also raises the possibility that the cytokine might have also affected this cell population. It is also conceivable that the unavoidable injury caused by cannula implantation (even after the several days of convalescence) may have promoted the activation of local inflammatory factors, thereby influencing the impact of subsequently administered IL-1 $\beta$ or IL-6. In this regard, it may be that surgical injury sensitized inflammatory processes, just as immune (LPS) or traumatic insults (stroke) had long term consequences upon neuroinflammatory responding. $5,49,50$

\section{Conclusions and future directions}

The present findings suggest that central and systemic administration of proinflammatory cytokines influenced hippocampal cellular proliferation and that these effects were dependent upon chronicity and route of administration. Indeed, systemic but not central TNF- $\alpha$ reduced BrdU, supporting the notion that this cytokine impaired cellular proliferation by acting upon peripheral targets. Conversely, repeated central infusion of IL-6 increased labelling of BrdU and the microglial marker, CD11b, suggesting that chronically elevated hippocampal levels of the cytokine increased the proliferation of microglia and possibly other cells. It is important to consider that since mice were sacrificed 24 hours following BrdU administration, cells labelled with this mitotic marker were at a very immature stage of development and hence, it is possible that after more protracted intervals different results may have been obtained. In this regard, it is conceivable that the cytokine treatments might influence the maturation or long term survival of neurons and glial cells. Nevertheless, the present results indicate that cytokines differentially influenced the early stages of production and/or proliferation of cells of the dentate gyrus.

\section{Acknowledgments}

The technical assistance of M Zhang and G Mott is greatly appreciated. We also thank $\mathrm{H}$ Anisman for critical evaluation of the manuscript. S Hayley is a Canada Research Chair (Tier 2) in Neuroscience. This work was supported by funds awarded to S Hayley from the Natural Sciences and Engineering Research Council (NSERC) and Canadian Institutes of Health Research (CIHR) Canada, as well as a New Investigator Awards from NARSAD.

\section{References}

1. Dunn AJ. The role of interleukin-1 and tumor necrosis factor alpha in the neurochemical and neuroendocrine responses to endotoxin. Brain Res Bull. 1992;29:807-812.

2. Hayley S, Anisman H. Multiple mechanisms of cytokine action in neurodegenerative and psychiatric states: Neurochemical and molecular substrates. Curr Pharm Des. 2005;11:947-962.

3. Hopkins SJ, Rothwell NJ. Cytokines and the nervous system. I: Expression and recognition. Trends Neurosci. 1995;18:83-88.

4. Weigent DA, Carr DJ, Blalock JE. Bidirectional communication between the neuroendocrine and immune systems. common hormones and hormone receptors. Ann N Y Acad Sci. 1990;579:17-27.

5. Hayley S, Lacosta S, Merali Z, van Rooijen N, Anisman H. Central monoamine and plasma corticosterone changes induced by a bacterial endotoxin: Sensitization and cross-sensitization effects. Eur J Neurosci. 2001;13:1155-1165.

6. Anisman H, Merali Z. Anhedonic and anxiogenic effects of cytokine exposure. Adv Exper Med Biol. 1999;461:199-233.

7. Maier SF, Watkins LR. Cytokines for psychologists: Implications of bidirectional immune-to-brain communication for understanding behavior, mood, and cognition. Psychol Rev. 1998;105:83-107.

8. Shintani F, Nakaki T, Kanba S, Kato R, Asai M. Role of interleukin1 in stress responses. A putative neurotransmitter. Mol Neurobiol. 1995;10:47-71.

9. Anisman H, Hayley S, Turrin N, Merali Z. Cytokines as a stressor: Implications for depressive illness. Int $J$ Neuropsychopharmacol. 2002;5:357-373.

10. Bluthé RM, Dantzer R, Kelley KW. Central mediation of the effects of interleukin-1 on social exploration and body weight in mice. Psychoneuroendocrinology. 1997;22:1-11.

11. Hayley S, Brebner K, Lacosta S, Merali Z, Anisman H. Sensitization to the effects of tumor necrosis factor-alpha: Neuroendocrine, central monoamine, and behavioural variations. J Neurosci, 1999;19:5654-5665.

12. Besedovsky HO, del Rey A, Klusman I, Furukawa H, Monge Arditi G, Kabiersch A. Cytokines as modulators of the hypothalamus-pituitaryadrenal axis. J Steroid Biochem Mol Biol. 1991;40:613-618. 
13. Bluthé RM, Beaudu C, Kelley KW, Dantzer R. Differential effects of IL-1ra on sickness behavior and weight loss induced by IL-1 in rats. Brain Res. 1995;677:171-176.

14. Merali Z, Brennan K, Brau P, Anisman H. Dissociating anorexia and anhedonia elicited by interleukin-1beta: antidepressant and gender effects on responding for "free chow" and "earned" sucrose intake. Psychopharmacology. 2003;165:413-418.

15. Kim JJ, Diamond DM. The stressed hippocampus, synaptic plasticity and lost memories. Nature Rev Neurosci. 2002;3:453-462.

16. Bremner JD, Narayan M, Anderson ER, Staib LH, Miller HL, Charney DS. Hippocampal volume reduction in major depression. Am J Psychiatry. 2000;157:115-118.

17. Sheline YI, Gado MH, Kraemer HC. Untreated depression and hippocampal volume loss. Am J Psychiatry. 2003;160:1516-1518.

18. Sheline YI, Wang PW, Gado MH, Csernansky JG, Vannier MW. Hippocampal atrophy in recurrent major depression. Proc Natl Acad Sci U S A. 1996;93:3908-3913.

19. Caetano SC, Hatch JP, Brambilla P, et al. Anatomical MRI study of hippocampus and amygdala in patients with current and remitted major depression. Psychiatry Res. 2004;132:141-147.

20. Manji HK, Quiroz JA, Sporn J, et al. Enhancing neuronal plasticity and cellular resilience to develop novel, improved therapeutics for difficult-to-treat depression. Biol Psychiatry. 2003;53:707-742.

21. D'Sa C, Duman RS. Antidepressants and neuroplasticity. Bipolar Disord. 2002;4:183-194.

22. Jacobs BL. 2002. Adult brain neurogenesis and depression. Brain Behav Immun. 2002;16:602-609.

23. Jacobs BL, Praag H, Gage FH. Adult brain neurogenesis and psychiatry: A novel theory of depression. Mol Psychiatry. 2000;5:262-269.

24. Kempermann G. Regulation of adult hippocampal neurogenesis implications for novel theories of major depression. Bipolar Disord. 2002;4:17-33.

25. Pham K, Nacher J, Hof PR, McEwen BS. Repeated restraint stress suppresses neurogenesis and induces biphasic PSA-NCAM expression in the adult rat dentate gyrus. Eur J Neurosci. 2003;17:879-886.

26. Thomas RM, Hotsenpiller G, Peterson DA. Acute psychosocial stress reduces cell survival in adult hippocampal neurogenesis without altering proliferation. J Neurosci. 2007;27:2734-2743.

27. Santarelli L, Saxe M, Gross C, et al. Requirement of hippocampal neurogenesis for the behavioural effects of antidepressants. Science. 2003;301:805-809.

28. McEwen BS. Protection and damage from acute and chronic stress: Allostasis and allostatic overload and relevance to the pathophysiology of psychiatric disorders. Ann N Y Acad Sci. 2004;1032:1-7.

29. Cameron HA, Gould E. Adult neurogenesis is regulated by adrenal steroids in the dentate gyrus. Neuroscience. 1994;61:203-209.

30. Kaneko N, Kudo K, Mabuchi T, et al. Suppression of cell proliferation by interferon-alpha through interleukin-1 production in adult rat dentate gyrus. Neuropsychopharmacology. 2006;31:2619-2626.

31. Vallières L, Campbell IL, Gage FH, Sawchenko PE. Reduced hippocampal neurogenesis in adult transgenic mice with chronic astrocytic production of interleukin-6. J Neurosci. 2002;22:486-492.

32. Monje ML, Toda H, Palmer TD. Inflammatory blockade restores adult hippocampal neurogenesis. Science. 2003;302:1760-1765.

33. Ekdahl CT, Claasen JH, Bonde S, Kokaia Z, Lindvall O. Inflammation is detrimental for neurogenesis in adult brain. Proc Natl Acad Sci US A. 2003;100:13632-13637.

34. Banks WA. Cytokines, CVSs, and the blood-brain-barrier. In: Ader R, Felten DL, Cohen N (eds). Psychoneuroimmunology, V. 2. New York: Academic Press;2001. pp. 483-498.

35. Bret-Dibat JL, Bluthe RM, Kent S, Kelley KW, Dantzer R. Lipopolysaccharide and interleukin-1 depress food-motivated behavior in mice by a vagal-mediated mechanism. Brain Behav Immun, 1995;9:242-246.

36. Rivest S, Lacroix S, Vallieres L, Nadeau S, Zhang J, Laflamme N. How the blood talks to the brain parenchyma and the paraventricular nucleus of the hypothalamus during systemic inflammatory and infectious stimuli. Proc Soc Exper Biol Med. 2000;223:22-38.
37. Christie BR, Cameron HA. Neurogenesis in the adult hippocampus. Hippocampus. 2006;16:199-207.

38. Brebner K, Hayley S, Zacharko R, Merali Z, Anisman H. Synergistic effects of interleukin-1beta, interleukin-6, and tumor necrosis factoralpha: Central monoamine, corticosterone, and behavioural variations. Neuropsychopharmacology. 2000;22:566-580.

39. Zalcman S, Green-Johnson JM, Murray L, et al. Cytokine-specific central monoamine alterations induced by interleukin-1, -2 and -6 . Brain Res. 1994;643(1-2):40-49.

40. Cerami A. 1992. Inflammatory cytokines. Clin Immunol Immunopathol. 1992;62(1 Pt 2):S3-10.

41. Varfolomeev EE, Ashkenazi A. Tumor necrosis factor: an apoptosis JuNKie? Cell. 2004;116:491-497.

42. Iosif RE, Ekdahl CT, Ahlenius H, et al. Tumor necrosis factor receptor 1 is a negative regulator of progenitor proliferation in adult hippocampal neurogenesis. J Neurosci. 2006;26:9703-9712.

43. Lacosta S, Merali Z, Anisman H. Influence of interleukin-1 on exploratory behaviors, plasma ACTH and cortisol, and central biogenic amines in mice. Psychopharmacology. 1998;137:351-361.

44. Juric DM, Carman-Krzan M. Interleukin-1 beta, but not IL-1 alpha, mediates nerve growth factor secretion from rat astrocytes via type I IL-1 receptor. Int J Dev Neurosci. 2001;19:675-683.

45. Gadient RA, Otten UH. Interleukin-6 (IL-6)--a molecule with both beneficial and destructive potentials. Prog Neurobiol. 1997;52:379-390.

46. Hirota H, Kiyama H, Kishimoto T, Taga T. Accelerated nerve regeneration in mice by upregulated expression of interleukin (IL) 6 and IL-6 receptor after trauma. J Exper Med. 1996;183:2627-2634.

47. Loddick SA, Turnbull AV, Rothwell NJ. Cerebral interleukin-6 is neuroprotective during permanent focal cerebral ischemia in the rat. J Cereb Blood Flow Metabol. 1998;18:176-179.

48. März P, Cheng JG, Gadient RA, et al. Sympathetic neurons can produce and respond to interleukin 6. Proc Natl Acad Sci U S A. 1998;95:3251-3256.

49. Barone FC, Parsons AA. Therapeutic potential of anti-inflammatory drugs in focal stroke. Exp Opin Invest Drugs. 2000;9:2281-2306.

50. Rosenzweig HL, Lessov NS, Henshall DC, Minami M, Simon RP, Stenzel-Poore MP. Endotoxin preconditioning prevents cellular inflammatory response during ischemic neuroprotection in mice. Stroke. 2004;35:2576-2581.

51. Goshen I, Kreisel T, Ben-Menachem-Zidon O, et al. Brain interleukin-1 mediates chronic stress-induced depression in mice via adrenocortical activation and hippocampal neurogenesis suppression. Mol Psychiatry. 2008;13:717-728

52. Vallières L, Rivest S. Regulation of the genes encoding interleukin-6, its receptor, and gp130 in the rat brain in response to the immune activator lipopolysaccharide and the proinflammatory cytokine interleukin-1beta. J Neurochem. 1997;69:1668-1683.

53. Fee D, Grzybicki D, Dobbs M, et al. Interleukin 6 promotes vasculogenesis of murine brain microvessel endothelial cells. Cytokine. 2000;12:655-665.

54. Szelényi J. Cytokines and the central nervous system. Brain Res Bull. 2001;54:329-338.

55. Rivest $\mathrm{S}$. How circulating cytokines trigger the neural circuits that control the hypothalamic-pituitary-adrenal axis. Psychoneuroendocrinology. 2001;26:761-788.

56. Banks WA, Kastin AJ, Broadwell RD. Passage of cytokines across the blood-brain barrier. Neuroimmunomodulation. 1995;2:241-248.

57. Worrall NK, Chang K, Lejeune WS, et al. TNF- $\alpha$ causes reversible in vivo systemic vascular barrier dysfunction via NO-dependent and independent mechanisms. Am J Physiol. 1997;273:H2565-H2574.

58. Laflamme N, Rivest $\mathrm{S}$. An essential role of interleukin- $1 ß$ in mediatong NF-KB activity and COX-2 transcription in cells of the blood-brain barrier in response to a systemic and localized inflammation but not during endotoxemia. $J$ Neurosci. 1999;19:10923-10930.

59. Wu JP, Kuo JS, Liu YL, Tzeng SF. Tumor necrosis factor-alpha modulates the proliferation of neural progenitors in the subventricular/ ventricular zone of adult rat brain. Neurosci Lett. 2000;292:203-206. 